\title{
Analysis of implementation of global nutrition policies to tackle the double burden of malnutrition in Nigeria, Zambia and Indonesia
}

\section{Abstract}

The double burden of malnutrition (DBM) is described as the co-existence of undernutrition and overweight or diet-related noncommunicable diseases within a country, community or an individual (WHO). It is a major global issue, affecting approximately half of the worlds' population. International organizations have published policy reports to function as guidelines for national implementation to tackle the double burden of malnutrition. The goal of this study is to analyze the advancement of the implementation of these recommendations in a number of middle-income countries.

Reports and recommendations on policies against DBM from 10 leading international organizations are analyzed and compared on several aspects, such as proposed measures, stakeholder involvement and target population. These organizations are UN, FAO, WHO, EAT forum, GAIN, World Food Program, World Bank, UNICEF, IFPRI and Global Panel on Agriculture and food systems for Nutrition. The level of implementation of the described policies is studied for Nigeria, Zambia and Indonesia by means of desk research and stakeholder interviews. These (semi-structured) interviews are based on the encountered information gaps within the desk research. Stakeholders were selected from a variety of organizations, such as government, NGO's, consumer organizations, science and industry in order to be able to examine the subject from different angles.

The study is still ongoing at the time of abstract submission. The (preliminary) results and conclusions will be presented and they are expected to describe per country which nutrition policies against DBM are initiated or implemented. Major learnings and best practices of these implementations will be reported and major differences and similarities between implementations in Nigeria, Zambia and Indonesia will be described and compared with the policy guidelines from the leading health organizations. These results may be used as learning when policies against DBM are newly implemented in other middle-income countries or to improve existing policies.

\section{Conflict of Interest}

There is no conflict of interest. 